\title{
Chronic prostatitis alters the prostatic microenvironment and accelerates preneoplastic lesions in C57BL/6 mice
}

\author{
Yong Gao ${ }^{1 \dagger}$, Lijuan Wei ${ }^{2 \dagger}$, Chenbang Wang ${ }^{2 \dagger}$, Yuanjie Huang ${ }^{2}$, Weidong $\mathrm{Li}^{2}$, Tianyu Li ${ }^{3}$, Chaohua Mo ${ }^{2}$,

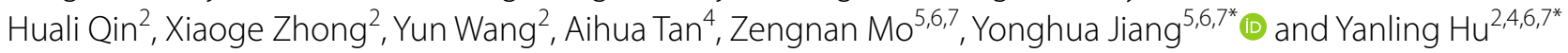

\begin{abstract}
Background: Chronic prostatitis has been supposed to be associated with preneoplastic lesions and cancer development. The objective of this study was to examine how chronic inflammation results in a prostatic microenvironment and gene mutation in C57BL/6 mice.
\end{abstract}

Methods: Immune and bacterial prostatitis mouse models were created through abdominal subcutaneous injection of rat prostate extract protein immunization (EAP group) or transurethral instillation of uropathogenic E. coli 1677 (E. coli group). Prostate histology, serum cytokine level, and genome-wide exome (GWE) sequences were examined 1, 3, and 6 months after immunization or injection.

Result: In the EAP and E. coli groups, immune cell infiltrations were observed in the first and last months of the entire experiment. After 3 months, obvious proliferative inflammatory atrophy (PIA) and prostatic intraepithelial neoplasia (PIN) were observed accompanied with fibrosis hyperplasia in stroma. The decrease in basal cells (Cytokeratin (CK) $5+/ p 63+)$ and the accumulation of luminal epithelial cells (CK8+) in the PIA or PIN area indicated that the basal cells were damaged or transformed into different luminal cells. Hic1, Zfp148, and Mfge8 gene mutations were detected in chronic prostatitis somatic cells.

Conclusion: Chronic prostatitis induced by prostate extract protein immunization or E. coli infection caused a reactive prostatic inflammation microenvironment and resulted in tissue damage, aberrant atrophy, hyperplasia, and somatic genome mutation.

Keywords: Chronic prostatitis, Prostatitis mouse model, Proliferative inflammatory atrophy, Prostatic intraepithelial neoplasia, Somatic genome mutation

\section{Introduction}

Prostate cancer is one of the most common cancers and the leading cause of cancer in the male reproductive system worldwide. The International Agency for Research on Cancer reported that prostate cancer is the second most frequent cancer among males in 2018 [1]. Effective treatments for prostate cancer include radical

\footnotetext{
*Correspondence: jiangyonghua@126.com; ylhupost@163.com

${ }^{\dagger}$ Yong Gao, Lijuan Wei and Chenbang Wang should be regarded as joint first authors

${ }^{7}$ Guangxi Collaborative Innovation Center for Genomic and Personalized Medicine, Nanning 530021, Guangxi, China

Full list of author information is available at the end of the article
}

prostatectomy, radiotherapy, and androgen deprivation therapy. However, about $15 \%$ of patients experience local recurrence or develop metastatic tumors [2]. If prostate cancer develops into castration-resistant prostate cancer, the treatment will be limited [3, 4]. Therefore, determining the key biological factors that lead to prostate cancer is important in establishing preventive treatments to halt the development of this cancer.

Prostatitis is a painful condition that involves the inflammation of the prostate and sometimes the areas around the prostate. Prostatitis can be divided into four types according to the prostatitis diagnosis guideline, and these types are chronic prostatitis/chronic pelvic pain

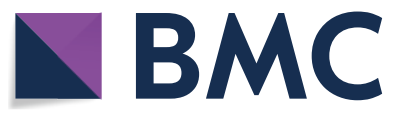

(c) The Author(s) 2019. This article is distributed under the terms of the Creative Commons Attribution 4.0 International License (http://creativecommons.org/licenses/by/4.0/), which permits unrestricted use, distribution, and reproduction in any medium, provided you give appropriate credit to the original author(s) and the source, provide a link to the Creative Commons license, and indicate if changes were made. The Creative Commons Public Domain Dedication waiver (http://creativecommons.org/ publicdomain/zero/1.0/) applies to the data made available in this article, unless otherwise stated. 
syndrome, acute bacterial prostatitis, chronic bacterial prostatitis, and asymptomatic inflammatory prostatitis [5]. Prostatitis is one of the most common prostate conditions in young and middle-aged men. The estimated prevalence of medically diagnosed prostatitis is $9 \%$, and the overall lifetime prevalence of prostatitis is estimated to be $14 \%$ [6]. The prevalence of NIH-IV prostatitis is $21.1 \%$ among 1868 asymptomatic men aged $19-78$ years and increases with age [7]. Nearly $20 \%$ of human cancers are associated with chronic inflammation [8]. Inflammation is closely related to prostate cancer initiation and progression [9]. Innate or adaptive immune cells or inflammatory cytokines can promote tumor initiation and progression. However, the inflammation in the pathogenesis of preneoplastic prostate lesions remains unclear.

In this study, we set up two mouse models for prostatitis induced by prostate extract protein immunization (EAP group) and E. coli infection (E. coli group). The characteristics of the inflammatory responses and proliferating prostate epithelial cells were evaluated, and the impact of chronic inflammation on the development of chronic pelvic pain was explored. In addition, gene mutation in the prostate was identified. This study helps us understand the role of prostate inflammation in the initiation and progression of prostate cancer.

\section{Materials and methods \\ Animals}

Experiments were performed on C57BL/6 mice that were approximately 9-11 weeks old and weighed 25-30 g (Chang Zhou Cavens Laboratory Animal Ltd., Nanjing, China). All animal procedures were approved and performed under the guidance of the Institutional Animal Care and Use Committee of Guangxi Medical University. The study was approved by Ethics and Animal Subject Committee of Guangxi Medical University.

\section{Induction of prostatitis}

For immune prostatitis, C57BL/6 male mice (9-11 weeks old) were injected subcutaneously in the abdominal flank with $200 \mu \mathrm{L}$ of an emulsion consisting of equal amounts of water and complete Freund's adjuvant (CFA, Sigma Aldrich, USA) with $100 \mu \mathrm{g}$ of prostate extract protein (EAP group). Control mice were immunized with CFA alone (CFA group).

For bacterial prostatitis, C57BL/6 male mice (9-11 weeks old) were anaesthetized (pentobarbital sodium, Merck, Germany) and intraurethrally instilled with $200 \mu \mathrm{L}$ of $E$. coli solution $\left(2 \times 10^{6} \mathrm{cfu}\right)$ (E. coli group). Control mice were instilled with sterility phosphate buffer saline (PBS) (PBS group).
For the naïve group, no special treatment was included (naïve group).

\section{Behavioral testing for the assessment of pain thresholds} At different time points of 1,3 , and 6 months after immunization or infection, the mice were tested for tactile allodynia and referred hyperalgesia by applying von Frey filaments to the pelvic region. All mice were tested in individual chambers with a stainless-steel wire grid bottom. Eight forces from 0.008 to $4 \mathrm{~g}$ were applied with different sizes of von Frey filaments. Positive behavioral responses included sharp retraction of the abdomen, instant licking, scratching, and jumping. The withdrawal response frequency was determined for each filament as the percentage of positive responses to 10 stimuli, each of which was applied for $3 \mathrm{~s}$ with a $5 \mathrm{~s}$ interval between stimuli. Data were expressed as the mean percentage of the response frequency for each filament.

\section{ELISA}

The serum level of $C$-reactive protein (CRP) was assessed as a nonspecific marker of inflammation and oxidative stress. CRP was measured using a Mouse C-Reactive Protein Quantizing ELISA kit (R\&D Systems, USA). High concentrations of cytokine IL-17 have been shown to promote prostate cancer progression in mice. IL-17 was measured by using a Mouse IL-17 Quantizing ELISA kit (R\&D Systems, USA). Absorbance was measured at $450 \mathrm{~nm}$ by using Varioskan Flash (Thermo Fisher Scientific, USA).

\section{Histology and Masson's trichrome staining}

The organs in the reproductive system, including prostate glands, testis, epididymis, and foreskin glands, were dissected. All organs were fixed in $10 \%$ buffered formalin overnight at $4{ }^{\circ} \mathrm{C}$ and embedded in paraffin. Sections $(5 \mu \mathrm{m}$ thick) were examined histologically through haematoxylin and eosin (H\&E) staining. Masson's trichrome staining was performed on prostate glands according to the Masson Stain Kit (Nanjing Jiancheng Bioengineering Institute, Nanjing, China).

\section{Immunohistochemistry}

Sections were deparaffinized, and antigen retrieval was performed by steam heating in $0.01 \mathrm{M}$ sodium citrate buffer ( $\mathrm{pH}$ 6.0) for $10 \mathrm{~min}$ in a steamer. Cooled slides were incubated with 5\% BSA blocking solution (Solarbio, Beijing, China) and incubated with primary antibodies overnight at $4{ }^{\circ} \mathrm{C}$. The primary antibodies included mouse anti-K5 (ab190083, Abcam, UK), rabbit anti-K8 (ab59400, Abcam, UK), rabbit anti-CD45 (ab10558, Abcam, UK), rabbit anti-Hsp60 (ab45134, Abcam, UK), and rabbit anti-p63 (ab735, Abcam, UK). For 
fluorescence visualization, the slides were incubated with secondary antibodies (diluted 1:300 in PBS containing $0.05 \%$ Tween 20$)$ and anti-rabbit $\lg G(\mathrm{H}+\mathrm{L})$ Fragment (Alexa Fluor 555 Conjugate) (Cell Signaling Technology, USA). Sections were counterstained with DAPI (Sigma Aldrich, USA). Immunofluorescence staining was imaged with a fluorescence microscope (Olympus BX53, Olympus Optical Co., Ltd.).

\section{Transmission electron microscopy}

The prostate dorsal lobe was cut into cubes of about $1 \mathrm{~mm}^{3}$, fixed with $3 \%$ glutaraldehyde (ALFA AESAR, USA) in $0.1 \mathrm{~mol} / \mathrm{L}$ PBS buffer ( $\mathrm{pH} \mathrm{7.4)}$ at $4{ }^{\circ} \mathrm{C}$ overnight, washed with PBS buffer three times, and post-fixed in 1\% osmium tetroxide for $1 \mathrm{~h}$ at room temperature. Afterward, the cubes were washed with PBS buffer and dehydrated in ethanol with an increasing concentration $(50 \%$, $70 \%, 90 \%$, and $100 \%$ ) in a sequential manner, followed by dehydration in a mixture of $90 \%$ ethanol and $90 \%$ acetone and then with $100 \%$ acetone three times for $10 \mathrm{~min}$ each. The tissue cubes were embedded in Spurr resin (SPI-CHEM, USA) and cut into thin slices (70-100 nm thickness) by using a microtome (Leica, EM UC7, USA). Samples were collected on 200-mesh copper grids and double stained with $1 \%$ aqueous uranyl acetate and $1 \%$ lead citrate aqueous solution (TED PELLA, USA). Then, the samples were examined under an H-7650 transmission electron microscope (HITACHI, Japan).

\section{DNA preparation and exome sequencing}

Genome-wide exome (GWE) sequencing was performed at Annoroad Gene Technology. Briefly, $0.6 \mu \mathrm{g}$ of genomic DNA per sample was used as the input material for DNA sample preparation. Sequencing libraries were generated with the Agilent SureSelect Human All Exon kit (Agilent Technologies, CA, USA) following the manufacturer's recommendations. Index codes were added to each sample. Complexes of library oligos and DNA fragments were captured, purified, and enriched via polymerase chain reaction (PCR). The DNA libraries were sequenced on an Illumina Hiseq platform, and $150 \mathrm{bp}$ paired-end reads were generated. Valid sequencing data were mapped to mm10 (https://www.sanger.ac.uk/science/data/mouse -genomes-project) by using the Bwa (bwa) + SAMtools (samtools) + RSeQC [10] to obtain the original mapping results stored in a binary alignment map (BAM) format.

\section{GWE sequence analysis work flow}

GATK (GenomeAnalysisTK.jar) were used to perform variant calling and identify SNP and InDels. Functional annotation is crucial because the link between genetic variations and diseases is clarified in this step. ANNOVAR software was applied to perform annotation on the variant call format (VCF) obtained previously. mm10 (refGene file) were used to characterize the detected variants. Annotation content contained the variant position, variant type, heatmaps depicting the total variant numbers per strain and the sum of strain-specific variants per two strain combinations were created in $\mathrm{R}$ by using [ggplot2] and [gridExtra] [11].

\section{Statistical analysis}

All experiments were performed independently using six mice. Data were presented as mean \pm standard error of the mean (SEM). Two-way analysis of variance and Student's $t$ test were used to determine the significance between groups. For all statistical tests, the 0.05 level of confidence was accepted for statistical significance.

\section{Result}

Immune injection or bacterial infection results in inflammation and immune reaction in the prostate

Prostate inflammation was evaluated histologically (H\&E-stained sections) and immunohistochemically. 1 to 6 months after treatment, significantly variable degrees of inflammation were observed among different groups and different prostate lobes (Fig. 1). A month after immunization or bacterial injection, the prostates of the mice in the naïve and control groups (CFA, PBS) showed normal prostatic histology, whereas immune cell infiltrations and significant inflammatory exudate in the glandular cavity were commonly observed in the EAP and E. coli groups. Inflammation occurred in the anterior and dorsolateral prostate lobes at high penetrance but less frequently in ventral prostate lobes. Immune cell infiltrations reached the peak on the third month. The CFA group exhibited very mild inflammation on the sixth month possibly due to the urinal reflex-induced chemical and physical trauma.

CD45 is a pan-hematopoietic marker of leukocytes [12, 13]. Immunofluorescence labeling for $C D 45$ revealed the immune cell distribution in the prostate lobes. Significant CD45 immunoreactivity was observed in the prostate lobes in the EAP and E. coli groups from 1 to 6 months after treatment (Additional file 1: Figure S1).

CRP, an acute phase protein, is assessed as a nonspecific marker of inflammation and oxidative stress [14]. The CRP in the EAP and E. coli groups was higher than that in the control and naïve groups 1 month after immunization or infection but not after 3 and 6 months (Fig. 2). The CRP serum levels in the EAP and E. coli groups increased a month after immunization or infection compared with the levels in the control and naïve groups. However, no statistically significant differences were observed between the EAP and E. coli groups for 1 month versus 3 months and 1 month versus 6 months. 


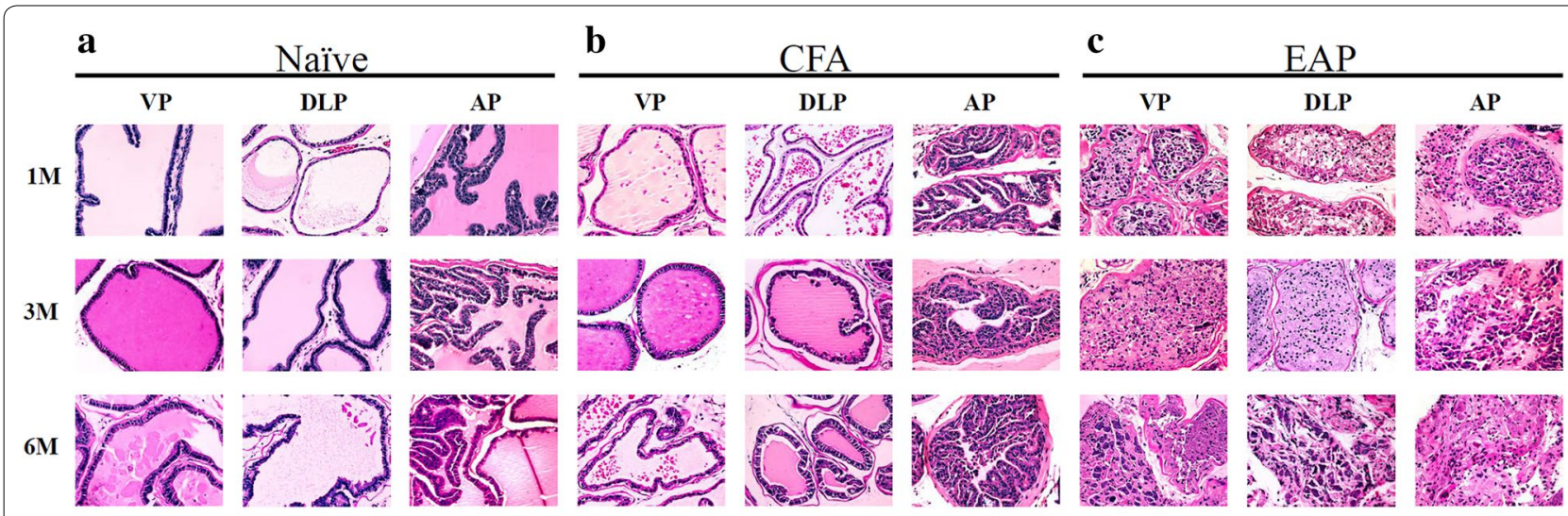

d

PBS

e

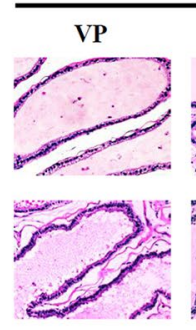

DLP

AP

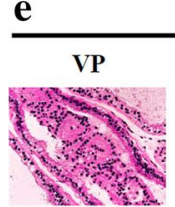

E. coli
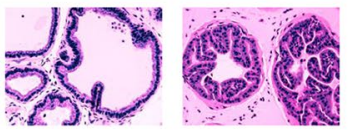

DLP

AP
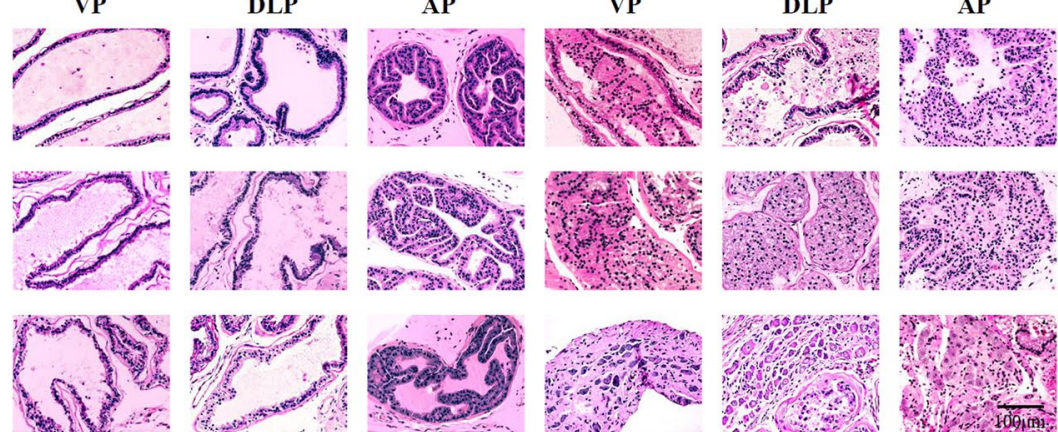

Fig. 1 Characteristic images of glandular histopathological findings in ventral prostate (VP), dorsolateral prostate (DLP), and anterior prostate (AP) lobes of mice at 1, 3, and 6 months after immunization or infection (H\&E stain, Bar $=100 \mu \mathrm{m}$ ). $\mathbf{a}, \mathbf{b}$, d Naïve, CFA, and PBS groups without obvious histopathological changes. CFA group with very mild inflammation at 6 months. c, e EAP and E. coli groups with inflammation characterized by infiltration of lymphocytes, hyperplasia and heteromorphism of glandular epithelium, large and intensely stained nucleolus, and karyokinesis

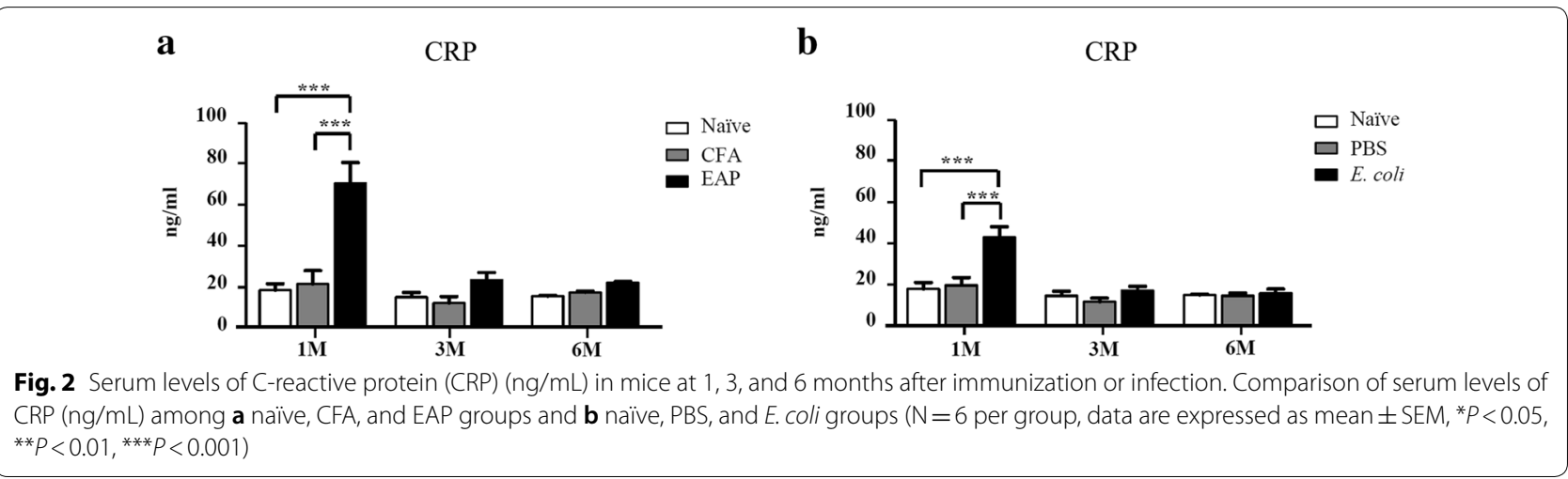

Likewise, no statistically significant difference was observed between the EAP and E. coli groups from one to 6 months. These results indicate a transformation from acute prostatitis to chronic prostatitis in the EAP and $E$. coli groups after immunization or infection.
Prostatitis was also manifested as an increased serum level of IL-17 (Fig. 3). IL-17 in the EAP group was much higher than that in the CFA group 3 and 6 months after immunization. The same trend was observed for the $E$. coli versus $\mathrm{PBS}$ versus naïve group. 
a

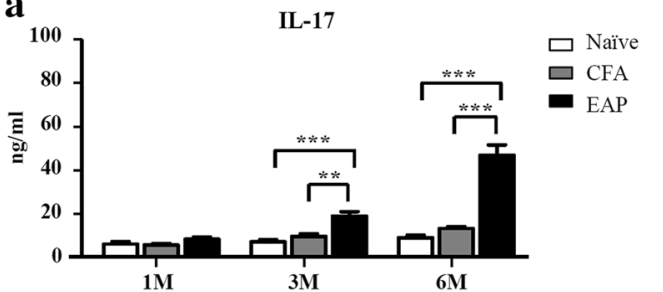

b

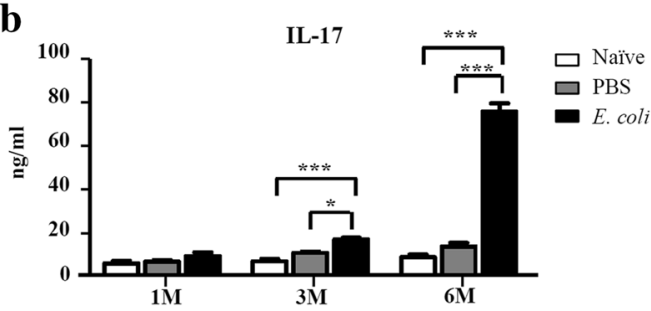

Fig. 3 Serum levels of IL-17 (ng/mL) in mice at 1, 3, and 6 months after immunization or infection. a Comparison of IL-17 (ng/mL) among naïve, CFA, and EAP groups. $\mathbf{b}$ Comparison of IL-17 ( $\mathrm{ng} / \mathrm{mL}$ ) among naïve, PBS, and E. coli groups ( $\mathrm{N}=6$ per group, data are expressed as mean $\pm \mathrm{SEM}$, $\left.{ }^{*} P<0.05,{ }^{* *} P<0.01,{ }^{* * *} P<0.001\right)$

\section{PIA and PIN were commonly observed in chronic prostatitis}

Compared with the control and naïve groups, glandular epithelial hyperplasia or atrophy was found in the EAP and E. coli groups from 3 to 6 months. PIN shows epithelial hyperplasia characteristics as the enlargement of the glandular lumen, hypertrophic cell, and irregular arrangement (Fig. 1, Additional file 2: Figure S2). PIA is recognized as the presence of atrophic epithelial cells, histological aggressiveness, loss or shrinking of acinar structures, focal atrophy lesions, dilated glandular lumens, and immature epithelial cells next to one another (Fig. 1, Additional file 2: Figure S2). In the high-magnification images, cells enriched with an intermediate phenotype that possessed properties of basal and luminal epithelial cells and debris composed of basal and glandular epithelial cells were found (Additional file 2: Figure S2).

CK5/CK8 immunofluorescence and p63 immunohistochemistry were applied to further distinguish the cell type (Figs. 4, 5). Basal cells with good cell plasticity display CK5 and p63, and functional luminal cells show the expression of CK8 [15]. Single layers of luminal cells $(\mathrm{CK} 8+)$ and basal cells $(\mathrm{CK} 5+/ \mathrm{p} 63+)$ were observed in the naivve and control groups. By contrast, a dramatic decrease in basal cells $(\mathrm{CK} 5+/ \mathrm{p} 63+)$ and accumulation of luminal epithelial cells (CK8+) were found in the inflamed prostatic lobes for EAP and E. coli groups. On the sixth month, nearly all of the CK5 stain disappeared in the EAP and E. coli groups (Fig. 5), which meant that the basal cells were damaged and transformed into different luminal cells.

Interstitial tissue hyperplasia was found, and Masson's trichrome staining showed an increase in collagen deposition (blue staining) and a reduction in smooth muscle cells (red staining) in the prostate stromal of the prostate lobes of EAP and E. coli groups from 3 to 6 months. Fibrosis increased with time (Additional file 3: Figure S3A). After quantification of the collagen fibers by Image Pro Plus 6.0 IOD, the EAP group showed a remarkable increase in the PBS group 3 and 6 months after treatment when compared with the CFA group (Additional file 3: Figure S3B). The same trend was observed for $E$. coli versus PBS or versus naïve group (Additional file 3: Figure S3C). Significant differences in muscle fibers were also noted among the EAP, CFA, and naïve groups from 1 to 6 months (Additional file 3: Figure S3D). Similar differences were found among the E. coli, PBS, and naive groups (Additional file 3: Figure S3E).

\section{Chronic prostatitis induced cell apoptosis}

We observed changes in the organelle ultrastructure through transmission electron microscopy. In the EAP and $E$. coli groups, changes in cellular organelles included swollen mitochondria with an irregular membrane, loss of mitochondrial membranes, disrupted mitochondrial granules, and irregular nuclear membranes. The CFA, PBS, and naïve groups showed a normal organelle ultrastructure, which indicates a tendency toward apoptosis in the EAP and E. coli groups (Additional file 4: Figure S4).

Heat shock protein 60 (HSP60) is a novel apoptotic and prostate cancer prognostic marker that is involved in the regulation of HIF- $1 \alpha$ protein stability and associated with prostate cancer progression [16, 17]. After prostate extract protein immunization or bacterial infection, the expression of HSP60 in the prostate lobes increased significantly from 1 to 6 months (Additional file 5: Figure S5A). Comparison of the IOD values of EAP, CFA, and naïve groups revealed significant differences (Additional file 5, Figure S5B). The same trend was apparent among E. coli, PBS, and naïve groups (Additional file 5: Figure $\mathrm{S} 5 \mathrm{C})$.

\section{Inflammatory prostate microenvironment caused chronic pelvic pain}

Referred hyperalgesia and tactile allodynia were examined by applying von Frey filaments to the pelvic region. The EAP and E. coli groups developed chronic pelvic pain, as indicated by decreased pain threshold responses to a stimulus in the pelvic region. Higher pain thresholds 


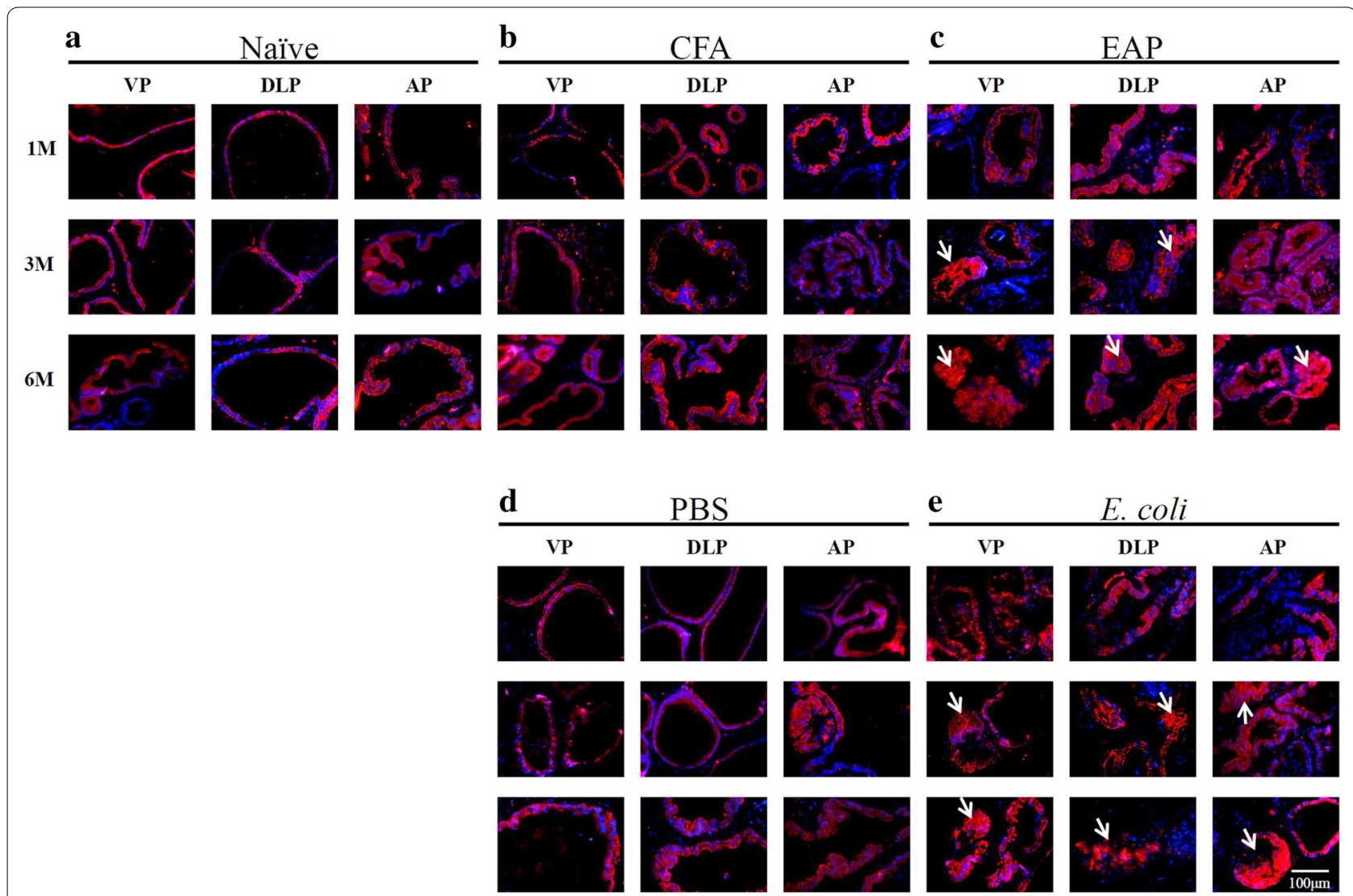

Fig. 4 Characteristic monochrome and merged images for CK8 (red) in ventral prostate (VP), dorsolateral prostate (DLP), and anterior prostate (AP) lobes of mice at 1, 3, and 6 months after immunization or infection. Nuclei are counterstained with DAPI (blue). $\mathbf{a}, \mathbf{b}, \mathbf{d}$ Naive, CFA, and PBS groups. A single layer of luminal cells is present, and no proliferating cells are observed. c, e EAP and E. coli groups. Multiple layers of luminal cells positive for CK8+ (in red) are observed at the third and sixth month of modeling

were observed in the EAP group compared with the CFA group. The same pattern was noted for the EAP versus naïve group (Additional file 6: Figure S6A). A similar trend was found for the $E$. coli group compared with the PBS group for 6 months (all $P<0.05$ ) (Additional file 6: Figure S6B).

To investigate how the inflammatory prostatic microenvironment influenced the histology of other organs in the reproductive system, H\&E staining was performed in the testis, epididymis, and foreskin gland of the mice in the EAP and E. coli groups 6 months after immunization or infection. The results showed variable degrees of inflammation in the testis, epididymis, and foreskin gland compared with the control and naïve groups (Additional file 7: Figure S7).

\section{Chronic inflammation resulted in somatic mutations}

The exon capture baits targeted $99.5 \%$ of genes in the GATK database mm10. Gene exon variants were filtered for quality according to several criteria (Additional file 8: Table S1). Variations were observed broadly across the genome in the naïve, EAP, CFA, E. coli and PBS groups at different time points. The mutation rate between different groups did not obviously differ. The gene exon variants ranged from 121 to 172 in different groups at different points, which included frameshift mutation, non-frameshift mutation, synonymous mutation, non-synonymous mutation, stop gain, and stop loss. The affected gene had no obvious difference in different groups (Additional file 9: Table S2, Additional file 10: Figure S8). We focused our analysis on genes with high or moderate impact variants, such as non-synonymous, frameshift mutation, stop gain, and stop loss, that are more likely to result in significant functional changes. As a result, several mutation genes were distinguished in the EAP or E. coli groups. Mutations affected the EAP group but were absent in the naïve or CFA group at 1 month (including Nacad, Prg4, and Zfp148), at 3 months (including Cyp3a41b, Glrp1, Gm572, Olfr849, and Sult2a6), and at six months (including Hic1 and Mfge8) (Table 1, Additional file 11: Table S3). Mutations affected the $E$. coli group but were absent in the naïve or PBS group at 


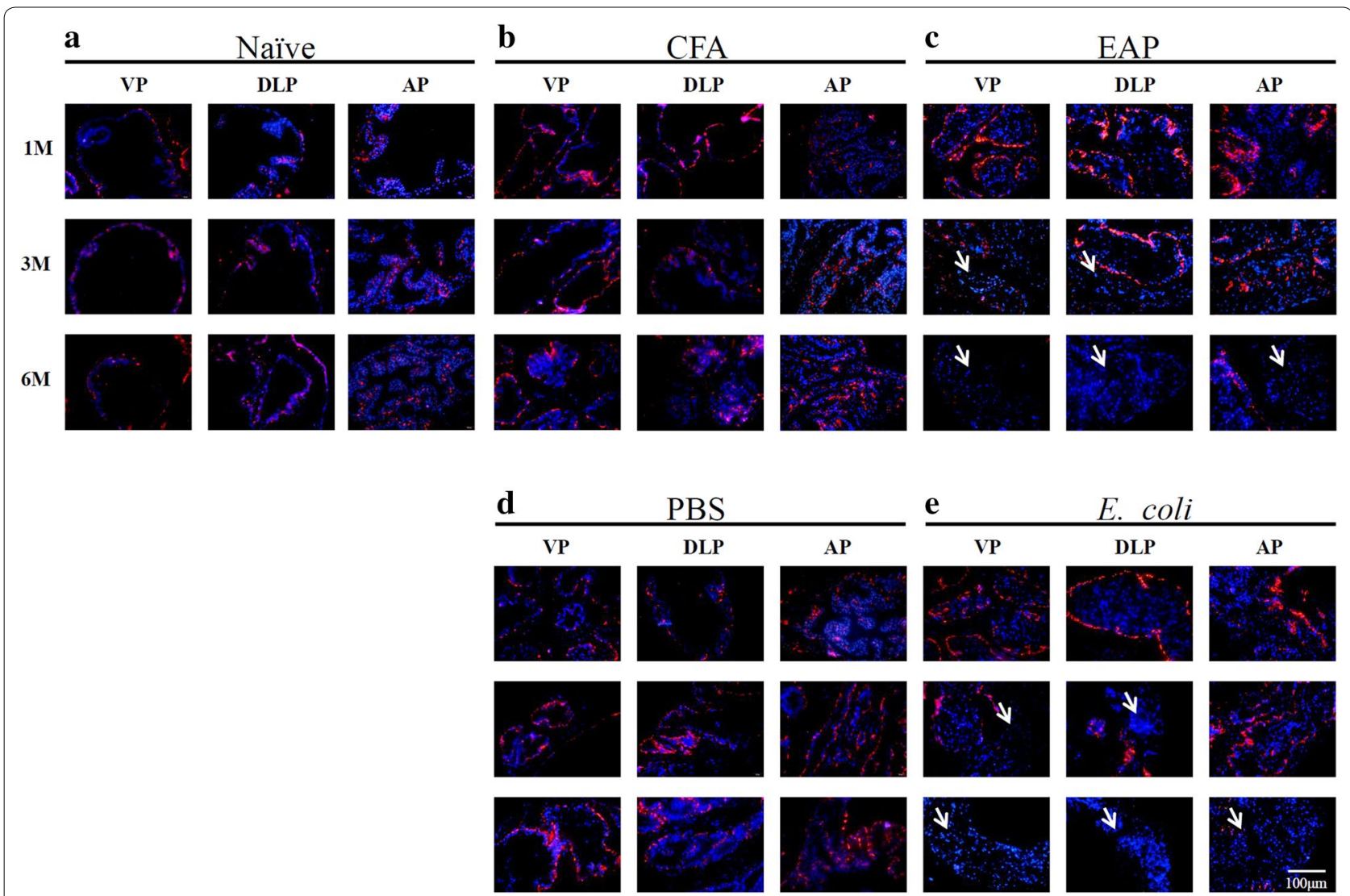

Fig. 5 Characteristic monochrome and merged images for CK5 (red) in ventral prostate (VP), dorsolateral prostate (DLP), and anterior prostate (AP) lobes of mice at 1, 3, and 6 months after immunization or infection. Nuclei are counterstained with DAPI (blue). $\mathbf{a}, \mathbf{b}, \mathbf{d}$ Naïve, CFA, and PBS groups. CK5+ (red) can be observed from 1 to 6 months. c EAP and E. coli groups. Basal cells positive for CK5+ (red) are observed in the first month, obviously decrease in the third month, and almost disappear in the sixth month

Table 1 Gene exon variants in EPA group but absent in CFA or naïve group

\begin{tabular}{llllll}
\hline & Chromosome & Gene & $\begin{array}{l}\text { EAP } \\
\text { (count) }\end{array}$ & CFA (count) & $\begin{array}{l}\text { Naïve } \\
\text { (count) }\end{array}$ \\
\hline 1 month & 11 & Nacad & 3 & 0 & 0 \\
& 1 & Prg4 & 8 & 0 & 0 \\
& 16 & Zfp148 & 3 & 0 & 0 \\
3 month & 5 & Cyp3a41b & 3 & 0 & 0 \\
& 1 & Glrp1 & 3 & 0 & 0 \\
& 4 & Gm572 & 3 & 0 & 0 \\
& 9 & Olfr849 & 3 & 0 & 0 \\
& 7 & Sult2a6 & 4 & 0 & 0 \\
& 11 & Hic1 & 3 & 0 & 0 \\
& 7 & Mfge8 & 3 & 0 & 0 \\
\hline
\end{tabular}

1 month (including Gm21708, Gm572, H2-Q6, Phactr4, Rsf1, Slc5a4b, and Zfp148), at 3 months (including Csprs, Gm572, and Slc5a4b), and at 6 months (including Cdh11, Mfge8, milk, and fat) (Table 2, Additional file 12:
Table S4). Beyond the gene difference, genomic variants in EAP and E. coli groups proved to be related. The most frequently mutated genes were Gm572, Slc5a4b, Zfp148, and Mfge8 (Additional file 13: Figure S9), which is genes that are recurrently mutated in chronic prostate inflammation.

\section{Discussion}

We established immune and bacterial prostatitis mouse models induced by prostate extract protein immunization injection or E. coli infection. CRP in the EAP and $E$. coli groups was higher than that in the control and naïve groups 1 month after immunization or infection. Serum IL-17 levels were elevated at 3 months and continued until 6 months. High inflammation cytokine helps strengthen the immune response in the initiation of inflammation, but long-term high concentrations of inflammation cytokines are harmful. Previous studies have shown that inflammatory cytokines, including CRP $[14,18]$, IL-6 [18-20], and IL-17 [21, 22], are positively associated with the risk of prostate cancer. Research has 
Table 2 Gene exon variants in EPA group but absent in CFA or naïve group

\begin{tabular}{llllll}
\hline & Chromosome & Gene & $\begin{array}{l}\text { E. coli } \\
\text { (count) }\end{array}$ & PBS (count) & $\begin{array}{l}\text { Naïve } \\
\text { (count) }\end{array}$ \\
\hline 1 month & $Y$ & Gm21708 & 3 & 0 & 0 \\
& 4 & Gm572 & 3 & 0 & 0 \\
17 & H2-Q6 & 6 & 0 & 0 \\
& 4 & Phactr4 & 3 & 0 & 0 \\
7 & Rsf1 & 3 & 0 & 0 \\
3 month & 1 & Slc5a4b & 3 & 0 & 0 \\
& 16 & Zfp148 & 3 & 0 & 0 \\
6 month & 8 & Csprs & 4 & 0 & 0 \\
& 10 & Gm572 & 3 & 0 & 0 \\
& 7 & Slc5a4b & 3 & 0 & 0 \\
\hline
\end{tabular}

proven that a high level of inflammation cytokine results in basal-to-luminal differentiation in mouse models [23]. Human and rodent basal cells possess the capacity for multiline age differentiation, and transfer of different basal cells is possible as preneoplastic lesions of prostate cancer [24-26].

Continuous inflammation leads to base membrane damage, epithelium atrophy, and abnormal proliferation $[9,15,27]$. In our present study, PIA and PIN were commonly observed in the EAP and E. coli groups after 3 months. Furthermore, a dramatic decrease in basal cells $(\mathrm{CK} 5+/ \mathrm{p} 63+)$ and accumulation of luminal epithelial cells $(\mathrm{CK} 8+)$ were found in the inflamed prostatic lobes for both EAP and $E$. coli groups, especially at the sixthmonth check point. This result means that the base cells were damaged or transformed into luminal cells. The PIA areas were enriched with cells phenotypically intermediate between basal and secretory cells. These cells expressed luminal epithelial cell marker CK8 and CK18, but they also expressed basal cell marker CK5 $[28,29]$. These cells exhibited high epithelial proliferation rates and infiltration by inflammatory cells [30]. PIA or PIN accumulated genetically changed lesions, such as GSTP1 hypermethylation [31-33] or MYC overexpression [34]. PIA lesions may give rise to carcinoma either directly or by developing into high-grade PIN [28, 31, 35]. In general, we think that chronic inflammation induced PIA and PIN cancer precursor lesions in this study, which are closely associated with prostate cancer.

Chronic pelvic pain is the most common symptom in chronic prostatitis patients. The basis of pelvic pain could be local neuroinflammation induced by local cytokine production from autoimmune/inflammatory processes $[36,37]$. Compared with the control and naïve groups, the EAP and E. coli groups showed significant increases in the pain threshold from 1 to 6 months. The phenomenon of prostatitis resulted in termed cross-organ sensitization. These findings are consistent with previous reports that inflammation in the prostate or bladder can induce the chronic prostatitis/chronic prostatic pain (CP/CPPP) syndrome [27]. The cross-organ sensitization symptom has also been observed in rat bladder inflammation models [38].

Prostate cancer is a tumor that is closely related to gene mutation. Common genetic alterations in prostate cancer include HPCI mutation [39], losses or downregulation of the tumor suppressor protein NKX3.1 [40, 41], loss or mutation of PTEN [42, 43], TMPRSS2-AR or ERG1-AR gene fusion [44-46], and AR upregulation [47]. Through GWE sequencing, we provided evidence that chronic inflammation induces somatic exon mutation in the prostate. Several mutation genes were distinguished in the EAP and CFA groups from the naïve, CFA, or PBS control groups. This is the first study to provide evidence that prostatitis induces somatic cell mutation in mouse models.

Mutations affected Hic1 in the EAP group but were absent in the naïve or CFA group at 6 months. This gene functions as a growth regulator and tumor repressor. Hypermethylation of $\mathrm{HIC} 1$ promoter results in the loss of its repressive function, and it is also a core gene mutation in prostate cancer [48]. Moreover, impairment of the HIC1 expression in the PCa cell could accelerates EMT (epithelial-mesenchymal transition) and promotes migration and metastasis. HIC1 rescue would significantly inhibit proliferation, migration, and invasion and induce apoptosis $[49,50]$.

Beyond the gene difference, genomic variants in the EAP and $E$. coli groups proved to be related. The most frequently mutated genes were Gm572, Slc5a4b, Zfp148, and Mfge8. Mfge8 is a bridge protein for membraneencased collection of proteins and triglycerides; it can facilitate efferocytosis and promote an innate immunity reaction by promoting macrophage polarization [51]. The serum level of Mfge8 is higher in prostate cancer patients than in control subjects [52].

Zfp148 is another gene mutation that came into notice. Zfp148 is a member of the Kruppel family of zinc finger DNA-binding proteins, and it interacts physically with the tumor suppressor protein 53 (P53). Zfp148 deficiency causes lung maturation defects and lethality in newborn mice, and this defect can be addressed by deleting Zfp148 binding P53 or providing antioxidant treatment [53]. Zfp148-deficient fibroblasts exhibit proliferative arrest [53]. Appropriate Zfp148 expression is important to cellular proliferation, embryogenesis, differentiation, growth arrest, and apoptosis [54]. 
To our knowledge, this study is the first to explore the roles of inflammation in prostate somatic gene mutation. However, this study has inevitable limitations. First, no prostate cancer outcome was observed, and only PIA and PIN were characterized. Second, exon sequences were used to detect somatic mutation in the prostate. Common mutations in prostate cancer, such as promoter hypermethylation, multi-copy gene segment, and gene fusion, might have been easily missed. Third, due to the lack of double staining CK8 and CK5, we could not distinguish intermediate cells that had properties of both basal and luminal epithelial cells in the PIA area.

\section{Conclusion}

Chronic prostatitis induced by prostate extract protein immunization or E. coli infection causes a reactive prostatic inflammation microenvironment and results in tissue damage, aberrant atrophy, hyperplasia, and somatic genome mutation. It also accelerates the initiation and progression of preneoplastic prostate lesions in C57BL/6 mice.

\section{Additional files}

Additional file 1: Figure S1. Characteristic images of immune cell distribution marked by CD45 immunolabeling (red fluorescence) in ventral prostate (VP), dorsolateral prostate (DLP), and anterior prostate (AP) lobes of mice at 1, 3, and 6 months after immunization or infection. A, B, D: Naïve, CFA, and PBS groups without immune cells at 1 month. CFA and PBS groups have few immune cells at 6 months. C, E: EAP and E. coli groups have immune cells in stroma in the beginning of modeling and even in the prostate glands 6 months after treatment.

Additional file 2: Figure S2. Characteristic monochrome and merged images for p63 in ventral prostate (VP), dorsolateral prostate (DLP), and anterior prostate (AP) lobes of mice at 1,3, and 6 months after immunization or infection. A, B, D: The naïve group has a single layer of basal cells, and no proliferating cells are observed. C, E: EAP and E. coli groups have multiple layers of basal cells positive for p63 in the beginning of modeling: the absence of basal cells in local prostate lobes appeared from 3 to 6 months after treatment.

Additional file 3: Figure S3. Masson's trichrome stain pictures of pathological fibrosis in ventral prostate (VP), dorsolateral prostate (DLP), and anterior prostate (AP) lobes of mice at 1, 3, and 6 months after immunization or infection. The red staining shows smooth muscle cells; the blue staining shows collagenous stroma. A: Naïve group without pathological fibrotic changes, CFA group without pathological fibrotic changes, EAP group with collagen deposition and dense fibrosis, PBS group without pathological fibrotic changes, and E. coli group with collagen deposition and dense fibrosis. Comparison of collagen fibers (average IOD in \%) among B: naïve, CFA, and EAP groups; C: naïve, PBS, and E. coli groups. Comparison of muscle fibers (average IOD in \%) among D: naïve, CFA, and EAP groups; E: naïve, PBS, and E. coli groups ( $N=6$ per group, data are expressed as mean \pm SEM, $\left.{ }^{*} P<0.05,{ }^{* *} P<0.01,{ }^{* * *} P<0.001\right)$.

Additional file 4: Figure S4. Transmission electron microscope images at 1, 3, and 6 months after immunization or infection. Naïve, CFA, and PBS groups without changes in the organelle ultrastructure. EAP and E. coli group with changes in the organelle ultrastructure, including disrupted mitochondrial granules, enlarged endoplasmic reticulum, degradation of mitochondrial cristae, accumulation of cytoplasmic lysosomes, and irregular nuclear membrane.

Additional file 5: Figure S5. Immunohistochemical Hsp60 expression in anterior prostate (AP), dorsolateral prostate (DLP), and ventral prostate (VP) lobes of mice at 1, 3, and 6 months after immunization or infection. A: Naïve, CFA, and PBS groups with no clear positive signals. EAP and E. coli groups with widespread strong positive signals in the cytoplasm. Comparison of Hsp60 (average IOD in \%) among B: naïve, CFA, and EAP groups and C: naïve, $P B S$, and $E$. coli groups ( $N=6$ per group, data are expressed as mean \pm SEM, $\left.{ }^{*} P<0.05,{ }^{* *} P<0.01,{ }^{* * *} P<0.001\right)$.

Additional file 6: Figure S6. Comparison of pelvic pain assessment among different groups. Proportion analysis (\%, left y axis) and average levels (right y axis) of pain thresholds in A: naive, CFA, and EAP groups and B: naïve, PBS, and E. coli groups ( $N=6$ per group, data are expressed as mean \pm SEM, $\left.{ }^{*} P<0.05,{ }^{* *} P<0.01,{ }^{* * *} P<0.001\right)$.

Additional file 7: Figure S7. Characteristic images of histopathological findings in the testis, epididymis, and foreskin gland of mice 6 months after immunization or infection (H\&E staining, Bar $=100 \mu \mathrm{m}$ ). Naïve, CFA, and PBS groups without histopathological changes. EAP and E. coli groups with inflammation characterized by infiltration of lymphocytes and hyperplasia.

Additional file 8: Table S1. SNP and index exclusion criteria.

Additional file 9: Table S2. Gene exon variants in CFA, EPA, E. coli, PBS and naive group at 1, 3, 6 month after injection or infection.

Additional file 10: Figure S8. Heat maps showing strain-specific profiles of the number of exome variants per 10-Mb bin across every chromosome in naive, CFA, EAP, PBS, and E. coli groups at 1, 3, and 6 months after immunization or infection. The darker the shade of blue is, the stronger the correlation is.

Additional file 11:Table S3. Mutations affected the EAP group but were absent in the naïve or CFA group at one, three and six months.

Additional file 12: Table S4. Mutations affected the E.coli group but were absent in the naïve or PBS group at one, three and six months.

Additional file 13: Figure S9. Venn diagram showing the overlap mutation gene among naïve, CFA, EPA, PBS, and E. coli groups at different time points of 1,3 , and 6 months.

\section{Abbreviations}

EAP: prostate extract protein; CFA: complete Freund's adjuvant; PBS: phosphate buffer saline; CRP: C-reactive protein; HSP60: heat shock protein 60; PIA: proliferative inflammatory atrophy; PIN: prostatic intraepithelial neoplasia; GWE: genome-wide exome; SEM: standard error of the mean.

\section{Acknowledgements}

Not applicable.

\section{Authors' contributions}

$\mathrm{YH}, \mathrm{ZM}$ conceived and designed the experiments. LW established mouse model of prostatitis. $Y H, W L, T L, C M$, behavioral testing of mouse. $H Q, X Z, Y W$, AT performed all of the pathology immunohistochemistry experiments. CW, WL performed bioinformatic analysis experiments. YG, YJ wrote the manuscript. All authors read and approved the final manuscript.

\section{Funding}

This research was supported in part by grants from the National Natural Science Foundation of China (Grant Numbers 81472414, 81360378, 81460388, 81770759), and the Guangxi Natural Science Foundation (Grant Numbers 2015GXNSFBB139008, 2018GXNSFAA138027).

\section{Availability of data and materials}

All data generated or analyzed during this study are included in this published article (and its additional information files). 


\section{Ethics approval and consent to participate}

Animal experiments conform to internationally accepted standards and have been approved by approved by the Ethics and animal Subject Committee of Guangxi Medical University.

\section{Consent for publication}

Not applicable.

\section{Competing interests}

The authors declare that they have no competing interests.

\begin{abstract}
Author details
${ }^{1}$ Department of Clinical Laboratory, The First Affiliated Hospital of Guangxi Medical University, Nanning 530021, Guangxi, China. ${ }^{2}$ Life Sciences Institute, Guangxi Medical University, Nanning 530021, Guangxi, China. ${ }^{3}$ Institute of Urology and Nephrology, First Affiliated Hospital of Guangxi Medical University, Nanning 530021, Guangxi, China. ${ }^{4}$ Department of Chemotherapy, The Affiliated Tumor Hospital of Guangxi Medical University, Nanning 530021, Guangxi, China. ${ }^{5}$ Center for Genomic and Personalized Medicine, Guangxi Medical University, Nanning 530021, Guangxi, China. ${ }^{6}$ Guangxi Key Laboratory of Genomic and Personalized Medicine, Nanning 530021, Guangxi, China. ${ }^{7}$ Guangxi Collaborative Innovation Center for Genomic and Personalized Medicine, Nanning 530021, Guangxi, China.
\end{abstract}

\section{Received: 21 March 2019 Accepted: 29 April 2019}

Published online: 14 May 2019

\section{References}

1. Bray F, Ferlay J, Soerjomataram I, et al. Global cancer statistics 2018: GLOBOCAN estimates of incidence and mortality worldwide for 36 cancers in 185 countries. CA Cancer J Clin. 2018;68(6):394-424.

2. DiBlasio CJ, Malcolm JB, Hammett J, et al. Survival outcomes in men receiving androgen-deprivation therapy as primary or salvage treatment for localized or advanced prostate cancer: 20-year single-centre experience. BJU Int. 2009;104(9):1208-14.

3. Ramsay AK, McCracken SR, Soofi M, et al. ERK5 signalling in prostate cancer promotes an invasive phenotype. Br J Cancer. 2011;104(4):664-72.

4. Maruyama Y, Miyazaki T, Ikeda K, et al. Short hairpin RNA librarybased functional screening identified ribosomal protein L31 that modulates prostate cancer cell growth via p53 pathway. PLoS ONE. 2014;9(10):e108743

5. Krieger JN, Nyberg L Jr, Nickel JC. NIH consensus definition and classification of prostatitis. JAMA. 1999;282(3):236-7.

6. Pontari MA, Joyce GF, Wise M, et al. Prostatitis. J Urol. 2007;177(6):2050-7.

7. Wu C, Zhang Z, Lu Z, et al. Prevalence of and risk factors for asymptomatic inflammatory (NIH-IV) prostatitis in Chinese men. PLoS ONE. 2013;8(8):e71298

8. Kundu JK, Surh YJ. Inflammation: gearing the journey to cancer. Mutat Res. 2008;659(1-2):15-30.

9. De Marzo AM, Platz EA, Sutcliffe S, et al. Inflammation in prostate carcinogenesis. Nat Rev Cancer. 2007;7(4):256-69.

10. Li H, Durbin R. Fast and accurate short read alignment with BurrowsWheeler transform. Bioinformatics. 2009;25(14):1754-60.

11. Gillespie A, Lee H, Robertson C, et al. Genome-wide exome analysis of Cmv5-disparate mouse strains that differ in host resistance to murine cytomegalovirus infection. G3. 2017;7(6):1979-84.

12. Deane JA, Ong YR, Cain JE, et al. The mouse endometrium contains epithelial, endothelial and leucocyte populations expressing the stem cell marker telomerase reverse transcriptase. Mol Hum Reprod. 2016;22(4):272-84.

13. Cai $Q$, Vethanayagam RR, Yang $S$, et al. Molecular profile of cochlear immunity in the resident cells of the organ of Corti. J Neuroinflamm. 2014;11:173.

14. Klyne DM, Barbe MF, Hodges PW. Systemic inflammatory profiles and their relationships with demographic, behavioural and clinical features in acute low back pain. Brain Behav Immun. 2017:60:84-92.

15. Shinohara DB, Vaghasia AM, Yu SH, et al. A mouse model of chronic prostatic inflammation using a human prostate cancer-derived isolate of Propionibacterium acnes. Prostate. 2013;73(9):1007-15.
16. Yang S, Meng J, Yang Y, et al. A HSP60-targeting peptide for cell apoptosis imaging. Oncogenesis. 2016;5:e201.

17. Ban HS, Shimizu K, Minegishi H, et al. Identification of HSP60 as a primary target of o-carboranylphenoxyacetanilide, an HIF-1alpha inhibitor. J Am Chem Soc. 2010;132(34):11870-1.

18. Shivappa N, Steck SE, Hurley TG, et al. Designing and developing a literature-derived, population-based dietary inflammatory index. Public Health Nutr. 2014;17(8):1689-96.

19. Tindall EA, Severi G, Hoang HN, et al. Interleukin-6 promoter variants, prostate cancer risk, and survival. Prostate. 2012;72(16):1701-7.

20. Boehm BJ, Colopy SA, Jerde TJ, et al. Acute bacterial inflammation of the mouse prostate. Prostate. 2012;72(3):307-17.

21. Zhang Q, Liu S, Ge D, et al. Interleukin-17 promotes formation and growth of prostate adenocarcinoma in mouse models. Cancer Res. 2012;72(10):2589-99.

22. Zhang Q, Liu S, Parajuli KR, et al. Interleukin-17 promotes prostate cancer via MMP7-induced epithelial-to-mesenchymal transition. Oncogene. 2017;36(5):687-99.

23. Kwon OJ, Zhang L, Ittmann MM, et al. Prostatic inflammation enhances basal-to-luminal differentiation and accelerates initiation of prostate cancer with a basal cell origin. Proc Natl Acad Sci USA. 2014;111(5):E592-600.

24. Goldstein AS, Huang J, Guo C, et al. Identification of a cell of origin for human prostate cancer. Science. 2010;329(5991):568-71.

25. Blackwood JK, Williamson SC, Greaves LC, et al. In situ lineage tracking of human prostatic epithelial stem cell fate reveals a common clonal origin for basal and luminal cells. J Pathol. 2011:225(2):181-8.

26. Park JW, Lee JK, Phillips JW, et al. Prostate epithelial cell of origin determines cancer differentiation state in an organoid transformation assay. Proc Natl Acad Sci USA. 2016;113(16):4482-7.

27. Haverkamp JM, Charbonneau B, Crist SA, et al. An inducible model of abacterial prostatitis induces antigen specific inflammatory and proliferative changes in the murine prostate. Prostate. 2011;71(11):1139-50.

28. De Marzo AM, Marchi VL, Epstein Jl, et al. Proliferative inflammatory atrophy of the prostate: implications for prostatic carcinogenesis. Am J Pathol. 1999;155(6):1985-92.

29. van Leenders GJ, Gage WR, Hicks JL, et al. Intermediate cells in human prostate epithelium are enriched in proliferative inflammatory atrophy. Am J Pathol. 2003;162(5):1529-37.

30. Goldman HB, Israeli RS, Lerner JL, et al. Effect of prostate biopsy on the results of the PSA RT-PCR test. Urology. 1998;52(6):1073-8.

31. Putzi MJ, De Marzo AM. Morphologic transitions between proliferative inflammatory atrophy and high-grade prostatic intraepithelial neoplasia. Urology. 2000;56(5):828-32.

32. Brooks JD, Weinstein M, Lin X, et al. CG island methylation changes near the GSTP1 gene in prostatic intraepithelial neoplasia. Cancer Epidemiol Biomarkers Prev. 1998;7(6):531-6.

33. Nakayama M, Bennett CJ, Hicks JL, et al. Hypermethylation of the human glutathione S-transferase-pi gene (GSTP1) CpG island is present in a subset of proliferative inflammatory atrophy lesions but not in normal or hyperplastic epithelium of the prostate: a detailed study using laser-capture microdissection. Am J Pathol. 2003;163(3):923-33.

34. Gurel B, Iwata T, Koh CM, et al. Nuclear MYC protein overexpression is an early alteration in human prostate carcinogenesis. Mod Pathol. 2008;21(9):1156-67.

35. Wang W, Bergh A, Damber JE. Morphological transition of proliferative inflammatory atrophy to high-grade intraepithelial neoplasia and cancer in human prostate. Prostate. 2009;69(13):1378-86.

36. Simons BW, Durham NM, Bruno TC, et al. A human prostatic bacterial isolate alters the prostatic microenvironment and accelerates prostate cancer progression. J Pathol. 2015;235(3):478-89.

37. Hu C, Yang H, Zhao Y, et al. The role of inflammatory cytokines and ERK1/2 signaling in chronic prostatitis/chronic pelvic pain syndrome with related mental health disorders. Sci Rep. 2016;6:28608.

38. Schwartz ES, La JH, Young EE, et al. Chronic prostatitis induces bladder hypersensitivity and sensitizes bladder afferents in the mouse. J Urol. 2016;196(3):892-901.

39. Smith JR, Freije D, Carpten JD, et al. Major susceptibility locus for prostate cancer on chromosome 1 suggested by a genome-wide search. Science. 1996;274(5291):1371-4. 
40. He WW, Sciavolino PJ, Wing J, et al. A novel human prostatespecific, androgen-regulated homeobox gene (NKX3.1) that maps to 8 p21, a region frequently deleted in prostate cancer. Genomics. 1997:43(1):69-77.

41. Bhatia-Gaur R, Donjacour AA, Sciavolino PJ, et al. Roles for Nkx3.1 in prostate development and cancer. Genes Dev. 1999;13(8):966-77.

42. Li J, Yen C, Liaw D, et al. PTEN, a putative protein tyrosine phosphatase gene mutated in human brain, breast, and prostate cancer. Science. 1997:275(5308):1943-7.

43. Yoshimoto M, Cutz JC, Nuin PA, et al. Interphase FISH analysis of PTEN in histologic sections shows genomic deletions in $68 \%$ of primary prostate cancer and $23 \%$ of high-grade prostatic intra-epithelial neoplasias. Cancer Genet Cytogenet. 2006;169(2):128-37.

44. Tomlins SA, Rhodes DR, Perner S, et al. Recurrent fusion of TMPRSS2 and ETS transcription factor genes in prostate cancer. Science. 2005;310(5748):644-8.

45. Carver BS, Tran J, Gopalan A, et al. Aberrant ERG expression cooperates with loss of PTEN to promote cancer progression in the prostate. Nat Genet. 2009;41(5):619-24.

46. King JC, Xu J, Wongvipat J, et al. Cooperativity of TMPRSS2-ERG with PI3-kinase pathway activation in prostate oncogenesis. Nat Genet. 2009;41(5):524-6.

47. Chen CD, Welsbie DS, Tran C, et al. Molecular determinants of resistance to antiandrogen therapy. Nat Med. 2004;10(1):33-9.

48. Kilinc D, Ozdemir O, Ozdemir S, et al. Alterations in promoter methylation status of tumor suppressor HIC1, SFRP2, and DAPK1 genes in prostate carcinomas. DNA Cell Biol. 2012;31(5):826-32.
49. Zheng J, Wang J, Sun X, et al. HIC1 modulates prostate cancer progression by epigenetic modification. Clin Cancer Res. 2013;19(6):1400-10.

50. Hao M, Li Y, Wang J, et al. HIC1 loss promotes prostate cancer metastasis by triggering epithelial-mesenchymal transition. J Pathol. 2017;242(4):409-20.

51. Raymond A, Ensslin MA, Shur BD. SED1/MFG-E8: a bi-motif protein that orchestrates diverse cellular interactions. J Cell Biochem. 2009;106(6):957-66

52. Soki FN, Koh AJ, Jones JD, et al. Polarization of prostate cancer-associated macrophages is induced by milk fat globule-EGF factor 8 (MFG-E8)mediated efferocytosis. J Biol Chem. 2014;289(35):24560-72.

53. Sayin VI, Nilton A, Ibrahim MX, et al. Zfp148 deficiency causes lung maturation defects and lethality in newborn mice that are rescued by deletion of p53 or antioxidant treatment. PLoS ONE. 2013;8(2):e55720.

54. Gao XH, Liu QZ, Chang W, et al. Expression of ZNF148 in different developing stages of colorectal cancer and its prognostic value: a large Chinese study based on tissue microarray. Cancer. 2013;119(12):2212-22.

\section{Publisher's Note}

Springer Nature remains neutral with regard to jurisdictional claims in published maps and institutional affiliations.
Ready to submit your research? Choose BMC and benefit from:

- fast, convenient online submission

- thorough peer review by experienced researchers in your field

- rapid publication on acceptance

- support for research data, including large and complex data types

- gold Open Access which fosters wider collaboration and increased citations

- maximum visibility for your research: over 100M website views per year

At BMC, research is always in progress.

Learn more biomedcentral.com/submissions 